Cultures \& Conflits

01 | hiver 1990

La prolongation des conflits

\title{
Le Nigéria : dynamique agonistique d'une Nation à polarisation variable
}

Guy Nicolas

\section{(2) OpenEdition}

1 Journals

Édition électronique

URL : http://journals.openedition.org/conflits/82

DOI : $10.4000 /$ conflits.82

ISSN : 1777-5345

Éditeur :

CCLS - Centre d'études sur les conflits lilberté et sécurité, L'Harmattan

Édition imprimée

Date de publication : 21 janvier 1990

ISSN : 1157-996X

Référence électronique

Guy Nicolas, «Le Nigéria : dynamique agonistique d'une Nation à polarisation variable », Cultures \& Conflits [En ligne], 01 | hiver 1990, mis en ligne le 30 décembre 2002, consulté le 30 mars 2021. URL: http://journals.openedition.org/conflits/82 ; DOI : https://doi.org/10.4000/conflits.82

Ce document a été généré automatiquement le 30 mars 2021.

Creative Commons License 


\title{
Le Nigéria : dynamique agonistique d'une Nation à polarisation variable
}

\author{
Guy Nicolas
}

1 La plupart des analyses contemporaines concernant les "constructions nationales" au sein des pays réputés en développement se basent sur un modèle centré sur le rôle fondateur quasi exclusif de l'État héritier du pouvoir colonial. Cette institution est censée concentrer sur elle la quasi-totalité de l'essence des sociétés soumises à sa gestion, même lorsqu'il est né d'un coup de force ou géré par des dirigeants ignorant les principes de l'état de droit qui lui sert de prototype. Ses activités s'exerceraient dans un vide social, table rase que sa mission consisterait à transformer progressivement en communauté nationale. Quant aux cadres sociaux hérités du passé, il s'agirait seulement de vestiges, responsables du sous-développement de ces pays. Tout au plus s'interroge-t-on sur la nature réelle des appareils que l'on désigne sous le nom d'État et sur les rapports qu'ils entretiennent avec la masse des citoyens, masse atomisée, soumise à des tendances centrifuges diverses.

Depuis quelques années, de tels modèles volent en éclat du fait de la ré-émergence d'identités mobilisatrices collectives dont on croyait qu'elles étaient en voie de résorption. Sous l'impact des explosions "tribales" ou "fondamentalistes" affectant de nombreux États, y compris des États considérés comme "développés", par exemple l'Union soviétique, les épures élaborées par les spécialistes des différentes "disciplines" concernées perdent leur efficacité, leur linéarité téléologique et fonctionnent de plus en plus comme des mythes incantatoires.

3 Devant cette situation, force est de reconnaître que les sciences sociales contemporaines ne disposent pas de modèles valables de l'État, de la nation, des sociétés "en développement", aussi bien que des réalités qu'occultent ces concepts. Une approche plus réaliste des données réelles devrait remettre en cause toutes les notions, tous les modèles élaborés à l'époque des indépendances lorsque le devenir du tiers monde était soumis aux chimères les plus utopiques qui ne survivent aujourd'hui que dans une ambiance de trompe-l'oeil généralisé. 
Une telle approche devrait prendre en compte la complexité souvent extrême des sociétés concernées, leurs contradictions, leurs dynamiques propres, les travestissements qu'y revêtent les modèles importés et la vitalité de polarisations collectives trop vite reléguées au rang d'anachronismes. Nous tenterons ici d'illustrer ces considérations par une brève esquisse de la dynamique nationale d'un pays qui compte près du tiers de la population du sous-continent noir : le Nigeria ${ }^{1}$.

Une société effervescente

5 La fédération nigériane appartient, comme le Brésil ou les Indes, au domaine des grandes nations très peuplées, bouillonnantes de vie et de contradictions, qui tranchent sur la grisaille des petits États écrasés sous la tutelle d'appareils clientélistes ou bureaucratiques figés et sur le chaos des nations impossibles, comme le Liban, le Soudan ou l'Ouganda. Les institutions les plus ordonnées y côtoient les foyers de violence. Les identités collectives les plus diverses et les plus contradictoires s'y bousculent dans un jeu perpétuel d'ajustements toujours provisoires. On y vit comme dans ces aires volcaniques où une éruption grave est toujours susceptible de survenir, mais où l'on s'accommode de cette menace. Les Cassandre qui prédisent périodiquement son explosion en se fondant sur l'évidence de tensions explosives sont constamment démentis par les "renouveaux" et autres situations perpétuant un consensus paradoxal.

6 Cette situation est liée à un substrat de crises plus profondes. En premier lieu, le "géant de l'Afrique" subit une croissance démographique de l'ordre de 3,5\% par an environ ${ }^{2}$. Sa population atteindrait aujourd'hui 112 millions d'âmes et pourrait s'élever à 280 millions en 2015. Cette seule donnée bouleverse de multiples cadres économiques, sociaux ou culturels. Elle est aggravée par une conjoncture économique non moins perturbatrice. Fondée à l'époque de l'indépendance sur une économie d'exportation de produits agricoles régionaux, elle a subi, au cours des années soixante-dix, les effets explosifs et ambigus d'un boom pétrolier qui a bouleversé ses bases. L'État fédéral, alors dominé par l'armée, en a profité pour construire une nouvelle économie entièrement fondée sur la redistribution d'une rente pétrolière importante et ses retombées, sur la base d'une articulation entre un secteur d'État dominant et un secteur privé affairiste et cosmopolite. L'ensemble de l'économie agricole antérieure s'est effondré. Les villes se sont développées de manière anarchique. Les bases productives générales de l'économie ont été négligées. La corruption s'est généralisée. Or, l'euphorie engendrée par cette croissance s'est brusquement brisée au début de la décennie actuelle, du fait de la crise de l'or noir et de l'endettement excessif de l'État. La fédération s'est réveillée d'un rêve de fortune et de grandeur pour se trouver en face d'une pénurie généralisée, du chômage, d'un écart croissant entre une minorité très riche et une masse de plus en plus appauvrie, ainsi que d'une criminalité croissante. Les mesures de redressement imposées par un nouveau gouvernement militaire porté au pouvoir par cette conjoncture ont aggravé cette situation. Sur le plan politique, la fédération a connu depuis l'indépendance deux républiques, deux régimes militaires, une grave guerre civile (sécession du Biafra), cinq coups d'État et de multiples crises. Le régime militaire qui s'est imposé en 1984 s'est engagé dans la mise sur rail progressive d'une troisième république, dont l'avènement est prévu pour 1992. L'ensemble de la classe politique civile antérieure est écartée de ce processus et le régime entend contrôler toutes les étapes de la transition, y compris la sélection d'une nouvelle élite politique (newbreed). Ces changements n'affectent pas la structure fédérale du pays, bâtie sur trois niveaux 
de gestion territoriale : fédéral, d'États fédérés et de "gouvernements locaux" laquelle a été fréquemment modifiée, les seconds ayant passé successivement du nombre de trois à quatre, douze, puis vingt et un, et les derniers de 301 à 449 , en 1989 , sans que ces remaniements répondent à toutes les exigences de la société.

La polarisation nationale

7 Le nationalisme nigérian est une réalité bien ancrée dans l'identité collective des habitants de la fédération ${ }^{3}$. II s'enracine, en premier lieu, dans l'attachement général au territoire hérité des découpages coloniaux. La majorité des Nigérians est attachée à l'intégrité de ce "pré carré", comme le montrent les mobilisations spontanées qui se produisent à chaque incident de frontière, même futile. Elle adhère aux mesures économiques liées à sa défense : monnaie nationale (naira), mesures protectionnistes, "nigérianisation" des firmes étrangères, centrage de l'économie sur la redistribution de la rente pétrolière, mesures d'austérité opposées aux ingérences du FMI... Elle s'identifie également aux symboles de souveraineté que sont le nom du Nigeria, son drapeau, son blason, son hymne national, sa Constitution. La fierté d'être nigérian s'appuie sur l'image d'un pays puissant, géant de l'Afrique, septième producteur d'or noir, cinquième plus grande démocratie du monde (en 1979-1983), "guide" et "grand frère" des autres pays monde (du continent, vouée à la haute mission messianique de défendre l'honneur de ce dernier et du monde noir dans son ensemble, y compris au sein de la diaspora sud et nord-américaine. Cette vocation messianique a conduit ses dirigeants, soutenus par les élites et une grande partie de l'opinion, à le poser en puissance régionale, capable de tenir tête aux plus grands. D'où les frustrations engendrées par sa situation d'isolat anglophone au sein d'un environnement presque exclusivement francophone et par l'influence $d$ autres puissances, même s'il arrive que les résistances à sa guidance ${ }^{4}$ soient étalées sur ses propres manifestations d'hégémonisme, comme il en a été à la suite d'expulsions massives d'immigrés d'autres pays africains ou dans le cadre de l'intervention de la force de l'Écowas au Liberia. Ce messianisme peut également déboucher sur un imaginaire de pays menacé dans sa stabilité par des adversaires résolus à réduire son rôle ou sa puissance, comme il en a été à l'occasion de ses prises de position en faveur de la ligne de front anti-apartheid. Tous ces thèmes sont largement débattus dans la presse locale, ainsi que dans les discours officiels de tous les gouvernements nigérians.

Ce patriotisme se nourrit de mythes historiques, tout autant que de l'attente d'un avenir radieux. Il met en relief la lutte toute relative pour l'indépendance, la "guerre civile" de 1967-1970, considérée comme un événement fondateur, et, surtout, le bref passage au pouvoir du général Murtala Mohammed, le "martyr", qui régna deux cents jours, de juillet 1975 à février 1976 et périt assassiné, après avoir conduit une politique marquée par son intégrité, le service de l'idéal national et une politique extérieure de prestige, illustrée par le défi porté aux Etats-Unis à propos de la reconnaissance du MPLA angolais. Cette haute figure est la référence obligée de toute entreprise patriotique. L'identité nationale nigériane se nourrit également d'une volonté de sauvegarde et de retour à un héritage ancestral "national" qui se définit surtout par opposition aux importations culturelles étrangères, pourtant omniprésentes. La mobilisation nationale peut se produire de manière spontanée, à l'occasion d'une agression frontalière, d'un succès international, y compris sur le plan sportif, d'un coup d'État populaire. Dans l'intervalle de ces processus spontanés, c'est toutefois l'État qui s'emploie à en entretenir la flamme. A l'indépendance du pays, c'est la monarchie (présidée par la reine d'Angleterre, en tant que reine du Nigeria) qui a maintenu les 
acquis de la construction coloniale, de 1960 à 1963. Sous la première République (1963-1966), le gouvernement fédéral du Premier ministre Tafewa Balewa s'est appliqué à poursuivre cette politique en dépit des effets centrifuges de la structure fédérale de la vie politique de l'époque et des accusations de ses adversaires de servir exclusivement les intérêts de sa région. Le coup d'État de janvier 1966, fomenté par une poignée de jeunes officiers "révolutionnaires", avait pour objectif de mettre un terme à une situation jugée par ceux-ci comme contraire à l'idéal national ${ }^{5}$. Mais ses adversaires l'ont perçu, au contraire, comme la manifestation d'un hégémonisme sudiste. Cette position s'est confortée sous le règne du général Ironsi, qui s'est substitué aux "majors". La stratégie jacobine de celui-ci, consistant à unifier l'espace national en brisant les barrières régionales, a suscité un mouvement de sécession nordiste débouchant sur l'éviction de ce gouvernement "sudiste". Porté par cette réaction, le colonel Gowon a dévié ce mouvement et s'est efforcé de rétablir l'autorité du pouvoir central et la cohésion du pays, en démembrant les anciennes entités régionales en douze États fédérés. Ces mesures ont provoqué la sécession de l'Eastern Region, sous le nom de Biafra. Le succès de cette entreprise eut engendré la balkanisation du pays. Mais l'appui des grandes puissances a permis au gouvernement fédéral d'écraser la "rébellion". Aussitôt, l'afflux de ressources pétrolières a permis à cet État de renforcer sa position, de former une nouvelle classe de bureaucrates fédéraux, de favoriser l'émergence d'une classe de businessmen attachés à l'entité nationale. Cette politique a été renforcée par le général Murtala Mohammed et son successeur, le général Obasanjo.

9 Sous la seconde République (octobre 1979 - janvier 1984), fondée sur une Constitution présidentielle et fédérale inspirée de celle des USA, le gouvernement du président Shagari s'est efforcé de maintenir l'autorité du gouvernement central malgré les stratégies et les critiques d'une opposition qui l'accusait de briser l'unité nationale. La crise qui s'est alors développée a conduit à un retour de l'armée, officiellement motivé par la nécessité de sauver une nation menacée dans ses fondements. Le général Buhari a mené une politique de consolidation du pouvoir central. Mais le caractère jugé trop rigoriste et autoritaire de sa gestion a conduit l'état-major à lui substituer le général Babangida, plus "ouvert"6. Ce dernier a conduit une politique économique rigoureuse. Mais il a engagé un nouveau processus de retour à un régime civil et démocratique étendu sur sept ans, écartant la classe politique antérieure, faisant élire une Constituante destinée à remodeler la Constitution de 1979, mais supprimant du texte nouveau les dispositions susceptibles, à ses yeux, de contrarier le processus et l'équilibre de la nouvelle république, écartant divers courants centrifuges ou porteurs d'autres projets (dont un projet socialiste). I1 suscita ainsi le retour à une vie politique partisane bipolaire dont les contours ont été étroitement délimités par ses soins, appelant les électeurs à une suite très étalée dans le temps d'élections aux diverses instances législatives et exécutives de la fédération, avant de transmettre ses pouvoirs à la nouvelle classe politique élue.

Dans cette évolution, l'armée nigériane a joué un rôle décisif. L'une des caractéristiques qui la distinguent de la plupart des pouvoirs prétoriens contemporains est de ne s'engager dans la voie politique qu'à son corps défendant, dans un dessein purement correctif- du moins le proclame-t-elle - et d'engager d'elle-même le processus de son retrait au profit d'un nouveau régime civil jugé plus démocratique et patriote que les précédents. Cette stratégie a été menée à bien de 1976 à 1979 et semble se renouveler aujourd'hui sous nos yeux de manière rigoureuse. Ce sont des militaires qui ont favorisé l'élaboration des deux Constitutions les plus démocratiques qu'ait connues le 
pays, alors que les deux régimes civils précédents ont sombré dans de graves crises politiques menaçant la cohésion de la nation. Ce sont également les militaires qui ont sauvé le pays du démembrement, en 1967-1970, et réussi un processus de réconciliation nationale. Et le chef d'État le plus incontesté de la fédération fut un militaire, à savoir le général Murtala Mohammed, symbole du dirigeant intègre, voué à l'unité nationale et à l'écoute de l'homme de la rue. On n'oubliera pas, cependant, que l'armée a connu de nombreuses crises internes, débouchant sur des putschs souvent très meurtriers, des séditions, des répressions fratricides, et qu'elle est d'autant plus à l'écoute des tensions qui affectent le pays que celles-ci menacent de la désintégrer elle-même de l'intérieur.

11 I1 est vrai que les régimes "militaires" sont également des appareils bureaucratiques, dominés par une fonction publique qui se considère comme le pilier de la construction nationale. Édifiée au lendemain de la guerre civile, à l'époque du boom pétrolier, celleci a bâti, effectivement, l'État central sur des bases solides notamment économiques, à l'encontre des aspirations des barons régionaux qui dominaient jusqu'alors le pays. Les technocrates ont toujours accusé les politiciens de préférer leurs intérêts à ceux de l'État. Mais ces derniers se veulent également les vrais bâtisseurs de la cohésion nationale. Beaucoup sont d'ailleurs issus de l'Administration. Le milieu d'affaires, né du boom économique, se considère également comme un support essentiel de l'idée nationale, d'autant plus que le recentrage de l'économie du pays sur l'unique rente pétrolière a brisé les bases sur lesquelles se fondaient les bourgeoisies régionales contrôlant les partis politiques de l'ère précédente. Le mouvement patriotique nigérian est également soutenu par les intellectuels, les universitaires, les écrivains, les milieux des médias (la presse nigériane est l'une des plus importantes et des plus libres du monde), la classe judiciaire, les syndicats, les étudiants, qui se considèrent comme l'avant-garde du patriotisme nigérian. On ne saurait sous-estimer également le rôle convergent des innombrables associations professionnelles, féminines, des clubs philanthropiques, des églises et autres clergés religieux, et même de la chefferie traditionnelle dans le développement d'un climat favorable aux grandes mobilisations patriotiques périodiques et à la promotion de l'idée nationale. Le régime militaire a renforcé ce dispositif par plusieurs institutions : Institut national d'études de politique et de stratégies (NIPSS), National Youth Corps, "collèges d'unité", institution du federal character, qui oblige en principe l'État à recruter ses agents sur une base de quotas par État fédéré.

12 Toutefois, le consensus national se trouve périodiquement mis en question du fait de l'existence de champs de polarisation concurrents, ordinairement refoulés à l'arrièreplan de la scène politique mais toujours susceptibles d'entrer en éruption et de mobiliser les citoyens sur des lignes centrifuges. La société nigériane se présente, en effet, comme un kaléidoscope constamment en mouvement où de grandes configurations concurrentes coexistent, se superposent, s'amalgament en mobilisant les mêmes sujets sur des positions diverses. Chacune de ces configurations de champs collectifs possède ses caractéristiques propres, ses valeurs, son discours, sa logique, ses contradictions. Chacune pourrait être au principe d'une élaboration nationale, à tel point que certains commentateurs pressés réduisent périodiquement cette société à l'un d'eux. Chacune constitue pourtant un champ spécifique qui a ses composantes particulières, ses lignes de rupture, ses traditions ou lois immuables, son "paysage social"

13 La contre-polarisation ethnique ${ }^{7}$ 
14 Selon un modèle courant, le Nigeria serait constitué de trois "nations" ethniques (hausa, igbo et yoruba). Cette perspective est fausse, puisque la fédération compte plus de deux cents formations de ce type. Il est vrai, cependant, que ces trois formations, appelées Big Three, Majors ou Wazobia ${ }^{8}$, dominent l'ensemble, du fait de leur importance (chacune compte de dix à trente millions de membres) et de leur position aux trois pôles géopolitiques du territoire national (le $\mathrm{Y}$ du blason nigérian). Ce tripode a dominé jusqu'ici la vie économique et politique du pays. Le champ ethnique s'est constitué à l'époque coloniale (aucune ethnie n'avait de cohérence sociopolitique auparavant). Il s'est trouvé renforcé du fait de la politique d'indirect rule britannique, des mouvements de population mettant en présence de la sociétés qui s'ignoraient, et des circonstances qui ont présidé à l'indépendance du pays. Le tripode dominant s'est assuré sur la base du dispositif économique, administratif et politique de cette époque, chaque formation luttant pour assurer son contrôle sur son fief régional et accéder aux postes de partage du gâteau national. L'expansionnisme de l'ethnie igbo, la plus scolarisée et la plus à l'étroit sur son territoire, a engendré la réaction défensive des autres grandes formations ${ }^{9}$. Ce conflit s'est stabilisé sur la base de la constitution par chacun des Big Three d'un fief régional d'où il s'est efforcé de chasser ses rivaux et qu'il a transformé en chasse gardée. Les aristocraties ou bourgeoisies de ces groupes dominants ont brisé les formations politiques nationales et mobilisé les membres de leurs groupements "tribaux" dans le cadre de partis politiques à fondement ethnique. Les minorités voisines, peu désireuses de subir l'hégémonie du "grand frère" local, se sont élevées contre ce mouvement, avec l'appui intéressé des grandes formations concurrentes de ce dernier. Toute la vie politique de la première République a été dominée par les déchirements résultant de cette situation.

Les auteurs du coup d'État de janvier 1966 avaient pour objectif avoué de briser celle-ci pour instaurer un nouvel ordre national. Mais cet événement a été perçu, au contraire, comme une manifestation d'hégémonisme igbo, de même que la politique jacobine du général Ironsi, qui s'est substitué aux Majors. D'où les pogroms anti-igbos en pays hausa et dans les casernes de la fédération, où les soldats ont massacré leurs officiers igbos. L'avènement du colonel Gowon a été perçue, au contraire, comme l'avènement d'un nouveau pouvoir émanant des minorités hostiles au pouvoir des trois grands. Quant à la sécession "biafraise", présentée par le colonel ojukwu comme une sécession régionale, elle fut considérée dans tout le reste de la fédération comme une tentative nationaliste de la seule ethnie igbo, déçue dans ses menées hégémoniques et traumatisée par les pogroms septentrionaux, vécus comme un génocide à son encontre ${ }^{10}$. D'où l'énergie des insurgés. Après la guerre civile, le gouvernement fédéral s'est employé à substituer aux mobilisations tribales l'idéologie nationaliste déjà évoquée, tout en accordant aux "minorités" des États distincts des fiefs des Big Three. La Constitution de 1979 a consacré cette politique. Mais les élections de 1979 ont été décevantes, dans la mesure où, si les Hausas se partageaient entre plusieurs partis, Igbos et Yorubas votaient massivement pour des partis qui leur étaient majoritairement spécifiques du fait de la présence à leur tête de grands leaders "tribaux" de l'époque de la première République: MM. Awolowo, pour l'LTPN, et Azikiwe pour le NPP Lors des élections suivantes (août 1983), la coalition, en principe majoritaire, de l'opposition au régime NPN a achoppé sur les conflits opposant en son sein les états-majors des deux partis en question au sujet de la priorité donnée à leurs leaders. Le parti national se divisait à son tour sur la question de la répartition des candidatures aux postes principaux du parti et de l'État entre quatre zones 
correspondant aux fiefs hausa, igbo, yoruba et des "minorités" méridionales. Le coup d'État militaire de janvier 1984 a brisé ce processus. Mais il a été perçu comme l'effet d'une stratégie hausa. L'éviction du général Buhari, membre de cette ethnie et son remplacement par le général Babangida, membre d'une minorité, en août 1985, a modifié à nouveau les données, certains de ses adversaires l'accusant cependant de favoriser la formation fulani-hausa ${ }^{11}$.

16 Au bloc divisé mais complice des Big Three s'oppose de plus en plus un autre ensemble constitué par toutes les autres ethnies ou "minorités" dont le point commun est de refuser l'hégémonie des premiers. Ainsi que nous l'avons vu, ces minorités mènent depuis l'indépendance une lutte destinée à échapper à leur satellisation par ceux-ci, bien qu'elles n'hésitent pas à s'allier avec les formations en question rivales de celle qui prétend exercer sur elles une hégémonie de fait. Il est vrai que cette situation se reproduit, au sein des États fédérés ou des local governments nouveaux où les Wazobia ne sont pas présents, entre "minorités" de plus ou moins grande importance, les dominés de la veille devenant souvent les hégémonistes locaux du lendemain. Mais c'est surtout à l'échelon fédéral que l'antagonisme évoqué se développe. Envenimé par le refus du colonisateur de démembrer les fiefs des Big Three au profit des aspirations à l'autonomie des minorités, il a débouché, sous la première République, sur la partition de l'Ouest en deux, la formation de partis minoritaires, dans l'Est et le Nord et l'insurrection de Tivs de la Benué. Le gouvernement du général Gowon, porté par ce nouveau bloc, a créé plusieurs États destinés à satisfaire leurs demandes d'émancipation à l'égard des "grands frères" voisins. La sécession biafraise, dressée contre cette stratégie, a achoppé sur la révolte des minorités de l'Eastern Region, déjà mobilisées dans le cadre d'un parti spécifique, celles-ci ne voyant dans le Biafra que l'instrument de la domination igbo. Elles ont alors rallié le camp fédéral, dont elles recevaient deux Etats, contribuant à l'isolement de la rébellion et à sa mise à l'écart des gisements pétroliers. Le bloc qu'elles constituent a été l'un des plus fermes soutien du mouvement national et du pouvoir fédéral. Sous la seconde République, la plupart des minorités ont voté pour le parti national contre les partis "tribaux" voisins. Certains changements de régime, comme l'avènement du colonel Gowon et celui du général Babangida, ont pu être perçus comme un fief de leur intervention. Ce bloc pourrait jouer un rôle majeur au cours de la troisième République, compte tenu du nombre d'États qu'il contrôle.

Chaque identité ethnique s'enracine dans un héritage culturel généralement reconstruit, mais effectivement mobilisateur. Celui-ci comprend une langue spécifique (d'où la complexité des débats sur le choix d'une langue nationale autre que l'anglais), un discours historique et mythique exaltant le modèle d'un peuple uni, l'attachement à des institutions particulières: chefferies locales, rituels, cérémonies traditionnelles fréquentées par les non-croyants, folklore, commémoration de grandes figures du passé. I1 s'étend aux vêtements usuels, au style de l'habitat, à divers types de comportements stéréotypés jugés caractéristiques de "l'héritage", dont certains se sont élaborés récemment dans les zones de confrontation entre groupes immigrés. Il supporte généralement des dispositions endogames et la persistance d'un népotisme ethnique obligeant beaucoup de citoyens à se classer, parfois contre leur gré, dans un cadre ethnique donné pour accéder à une place ou une promotion, ou défendre leurs droits. Mais le fondement premier des mobilisations ethniques demeure politique. Celles-ci se sont érigées en entités solidaires à l'époque des premiers partages partisans, les leaders politiques ayant alors tendance à chercher à se constituer des 
électorats fidèles en mobilisant les membres de leur "tribu" sur cette base et en agitant des arguments xénophobes à l'encontre des "étrangers" envahisseurs. Les partis de cette époque se sont édifiés sur le socle d'associations culturelles vouées à la défense et à la promotion d'ethnies particulières. Ces mobilisations ne sont toutefois jamais exclusives ni permanentes et succombent souvent devant les courants de division issus d'un passé où l'unité ethnique était toute relative, la concurrence d'autres pôles de mobilisation internes ou externes, les clivages nés d'affrontements de leaders locaux ou de confrontations partisanes. De telles divisions ont favorisé les stratégies des autres pouvoirs, et notamment celle du pouvoir central visant à briser les stratégies ethniques et à les transférer sur le pôle national. Il n'en reste pas moins que le partage ethnique demeure une source de division que des politiciens en quête d'électorat ou d'audience peuvent toujours chercher à raviver avec des succès variables, ou de mobilisations spontanées à l'occasion de problèmes de terre, de postes ou du meurtre de sujets appartenant à un groupe donné. II demeure également au principe de nombreuses revendications visant à de nouveaux remembrements de la structure fédérale du pays

La contre-polarisation régionaliste

Un second champ de polarisation concurrent du champ national repose sur le clivage du pays en trois aires géopolitiques régionales. Souvent confondu avec la configuration ethnique du fait de l'implantation et des visées hégémoniques des Big Three, il s'en distingue néanmoins nettement, ne serait-ce que parce qu'il associe d'emblée des ethnies différentes au sein d'ensembles constituant ses diverses composantes permanentes. Cette association s'effectue sous le chef de traditions socioculturelles transcendant les particularismes ethniques et formant des supra-cultures locales. Celles-ci peuvent correspondre à celle du groupe dominant. Mais il n'en est ainsi qu'à un niveau superficiel. Ainsi, le clivage régional de base de la fédération oppose deux ensembles septentrional et méridional. Le premier s'inscrit dans le cadre du vaste ensemble culturel formé par la culture "soudanaise", qui s'étend du cap Vert à la mer Rouge et le second dans celui d'une non moins vaste culture forestière ou côtière, tout aussi spécifique. Cette opposition s'est trouvée renforcée au nord par l'ouverture de la frange subsaharienne au commerce arabo-berbère et à l'influence islamique, qui a marqué les cultures locales dominantes, puis par le mouvement fondamentaliste musulman (jihad) du début du siècle dernier dont est né le sultanat de Sokoto.

Dominé par une aristocratie d'origine immigrante et pastorale, celui-ci a étendu son empire à un vaste espace s'étendant du Niger au Nord-Cameroun et de Katsina au sud d'Ilorin, influençant une grande partie des populations septentrionales, de manière positive ou négative. Simultanément, les sociétés côtières s'ouvraient à la traite européenne, au commerce maritime, puis à l'influence des missions chrétiennes, lesquelles ont utilisé d'anciens esclaves locaux convertis en exil et dont la préoccupation était de "civiliser" leurs proches. La politique coloniale britannique a renforcé cette opposition en dissociant deux "protectorats", septentrional et méridional, et en maintenant ce clivage sous la forme d'un traitement administratif différent, après l'association de ces deux territoires au sein d'une même entité "nigériane". En 1914, dans le Nord, qu'elle entendait soustraire à l'influence jugée délétère de la "civilisation", l'administration du gouverneur Lugard a favorisé le pouvoir des aristocraties musulmanes, la langue hausa, en tant que langue commune, l'islam, dissociant les élites septentrionales de celles du Sud et opposant des obstacles à la diffusion du christianisme et de la scolarisation. Dans le Sud, au contraire, une nouvelle élite christianisée, scolarisée, éprise de progrès s'est ouverte à l'influence 
occidentale, s'efforçant de faire "évoluer" les populations locales et d'obtenir le retrait du colonisateur. Ce sont des méridionaux qui ont constitué les premiers partis politiques nationalistes, le Nord demeurant jusqu'à l'indépendance dans un état d'arriération général. De nombreux méridionaux, notamment igbos, et plus particulièrement des educated, ont développé une nouvelle diaspora au sein de cette région, introduisant des idées qui gênaient aussi bien l'administration coloniale que l'aristocratie locale.

20 Pour contrer ce courant, le colonisateur s'est employé à accentuer l'isolement du Nord, refusant de diviser cette zone majoritaire au profit des revendications des minorités hostiles à l'hégémonisme hausa, formant une élite régionale distincte, accentuant l'expansion de la langue hausa et de l'islam. Parallèlement, ils s'employaient à diviser le Sud en favorisant le développement de conflits entre bourgeoisies igbo et yoruba. S'appuyant sur une répartition régionale de l'économie du pays, fondement d'une nouvelle distribution des ressources issues de la commercialisation de produits agricoles locaux, le colonisateur a enfin organisé la fédération sur la base de trois Régions dotées chacune d'une certaine autonomie. Les élites locales ont suivi le mouvement et constitué des partis régionaux organisés pour le contrôle de ces cadres territoriaux et l'accès au pouvoir central, issu des Regions: NPC pour la Northern Region, NCNC pour l'Eastern Region (Est du delta), Action Group pour la Western Region (Ouest du delta). Ce dispositif a brisé l'élan des premiers mouvements nationaux. Les deux partis méridionaux évoqués ici ont de plus en plus oscillé entre une politique d'union contre l'hégémonisme septentrional et une stratégie d'affrontement marquée par la confrontation de l'Est et de l'Ouest, l'alliance du NCNC et du NPC contre l'Action Group, l'éviction de celui-ci du contrôle du gouvernement de l'Ouest et du pouvoir central, l'entrée en rébellion de la Western Region et de graves tensions entre une alliance à dominante nordiste (NNA) et une autre à dominante sudiste (UPGA), elle-même divisée sur une base régionale. Cette situation a débouché sur le coup d'État "révolutionnaire" de janvier 1966.

Quelles qu'aient été les intentions des auteurs de celui-ci, il a été perçu au Nord comme une manifestation d'hégémonie sudiste autant qu'igbo. Il en a été de même de la politique du général Ironsi de démembrement du système fédéral, le Nord tout entier se mobilisant alors contre les "envahisseurs" sudistes et menaçant de faire sécession. Le contrecoup de juillet 1966 fut avant tout un mouvement nordiste à tendance partitionniste. Le colonel Gowon, qu'il a propulsé sur le devant de la scène, était un minoritaire septentrional, et non un Hausa, et une part importante des troupes nordistes était constituée de Tivs, alors en pleine rébellion contre l'hégémonisme de la formation considérée. En revanche, la sécession biafraise, qui a pris la relève de la menace partitionniste du Nord, ne correspondait pas à un nationalisme propre à l'ensemble de la région de l'Est, comme ont pu le croire ses supporters. En effet, les minorités de cette région, dressées contre l'hégémonisme igbo, ont immédiatement rallié le parti fédéral, privant le colonel ojukwu de ses arguments régionalistes et favorisant l'échec du Biafra. Sous la seconde République, les mêmes minorités, dotées d'États fédérés propres, ont voté majoritairement pour le parti national du président Shagari. Par contre, les électeurs de l'ancienne Western Region votaient unanimement pour l'UPN, avant de se diviser à l'occasion des élections de 1983. La coalition "progressiste", formée par l'UPN, le NPP et leurs alliés, s'est alors constituée sur les bases mêmes de l'UPGA de 1965-1966, achoppant sur les mêmes tensions entres "orientaux" et "occidentaux", tandis qu'Igbos et Yorubas se divisaient entre partisans 
du NPP ou de l'UPN et du NPN, accusé en 1979 de représenter le parti nordiste. Mais l'ouverture du parti national du président Shagari à des éléments méridionaux et minoritaires, conçue dans un but de mobilisation nationale, a suscité une scission de ce parti, déclenchée par les tenants les plus irréductibles d'une hégémonie proprement nordiste, regroupés dans la Kaduna mafia. C'est à ce groupe de pression qu'a été attribué par la suite le coup d'État de la Saint Sylvestre 1983, le gouvernement du général Buhari étant à dominante septentrionale. Son éviction, en août 1985, a été perçue, au contraire, comme une nouvelle intervention des minorités hostiles aux positions régionalistes qui les défavorisaient. Aujourd'hui, les actes de ce gouvernement sont fréquemment évalués en fonction des avantages qu'ils réservent au Nord ou au Sud, même si ces expressions ne correspondent plus à aucune réalité politico-administrative.

Les polarisations régionales sont cependant loin de constituer des alternatives permanentes et sérieuses au mouvement national. Nombreux sont les Nigérians qui refusent de se classer dans un camp régional ou combattent les positions en question. Par ailleurs, les espaces régionaux de l'ère de l'indépendance n'ont jamais été cohérents, la Northern Region se divisant en deux blocs antagonistes (Far North et Middle Belt) et, nous l'avons vu, le Sud en deux blocs oriental et occidental, le premier étant partagé entre un noyau igbo dominant et une périphérie minoritaire (COR) et le second entre un foyer yoruba et un autre ensemble, minoritaire, obtenant en 1963 une région propre, découpée dans l'ancienne Western Region: le Mid-West (devenu aujourd'hui l'État de Bendel). Mais les polarisations régionalistes continuent à commander à des polarisations culturelles, à des différences de comportement, d'état d'esprit, d'ouverture sur l'art, de solidarités spontanées et à des rejets collectifs. Elles supportent des stratégies de division que s'emploient à perpétuer divers groupes de pression attachés à ces particularismes et autres comités d'elders du Nord ou du Sud. Il est toujours possible, en effet, d'éveiller des sensibilités latentes en dénonçant des déséquilibres régionaux dans tous les domaines, notamment dans celui des affectations administratives ou partisanes, ou des mesures du gouvernement central jugées partiales, comme la fermeture de six universités méridionales, en réponse aux manifestations d'étudiants de juin 1989, un remaniement ministériel ou administratif, comme celui de décembre 1989 et le caractère sévère de la répression des tentatives de putsch dirigées contre l'équipe militaire au pouvoir, en 1976, 1986 et 1990. La dernière de ces tentatives, qui a été l'une des plus violentes qu'ait connu le pays depuis janvier 1966 et qui s'inscrit, d'ailleurs, dans la ligne de celle-ci, a vu un groupe non négligeable d'officiers et de soldats s'emparer de différents bâtiments publics, dont celui de la télévision et une partie du quartier général de Lagos, bombarder au canon la résidence du chef de l'État, dans le but de l'assassiner et proclamer une sécession du pays sur la base d'un clivage entre États du Far North et États du Sud et du Middle Belt, le 22 avril 1990. Si elle a échoué, le procès qui a suivi, comme les prises de parti de nombreux groupes de pression, objets d'une surveillance sévère de la part d'un régime pourtant engagé dans un processus de démocratisation, et l'exécution des principaux acteurs du putsch ont fait apparaître au grand jour la subsistance de tendances que l'on avait crues en déclin. Les conséquences de cet événement sur la cohésion de l'armée, les orientations du régime et les stratégies de la classe politique au sein des deux partis politiques autorisés, profondément divisés sur des bases régionalistes, semblent importantes, d'autant plus qu'elles ont rappelé aux élites nordistes le coup d'État meurtrier du 15 janvier 1966, qui décima leur état-major, institua une nouvelle ère de 
pouvoir à base militaire, suscita la sécession biafraise et favorisa la montée de forces politiques nouvelles. L'interdiction, en septembre 1990, d'une conférence-débat sur le thème de la question nationale nigériane par un gouvernement attaché à donner de luimême une image libérale s'explique par le souci de ce dernier d'éviter la ré-émergence de revendications exclues de son programme de retour à un régime démocratique élu pour 1992.

(1) essionnelles, compte tenu de la charge passionnelle qu'elles détiennent, le Nigeria comptait alors $47,2 \%$ de musulmans, $34,5 \%$ de chrétiens et I8,3\% d'adeptes de cultes ancestraux. Aujourd'hui, l'OCI avance le pourcentage de $70 \%$ de musulmans, tandis que les Églises proclament que leurs adeptes sont majoritaires, les leaders de ces cultes "importés" spéculant peut-être trop vite sur la disparition des religions africaines. Un modèle usuel, fondé sur le schéma régionaliste et promu par le pouvoir colonial, divise la fédération en un Nord musulman et un Sud chrétien et animiste. En réalité, une partie importante des septentrionaux n'adhère pas à l'islam, et une forte proportion de Méridionaux, à l'exception de ceux qui vivent à l'est du delta, se range sous les bannières de l'islam. Les musulmans sont majoritaires dans les trois États d'Ogun, de Lagos et d'Oyo. Si le modèle géo-religieux évoqué correspond à quelque réalité, celle-ci est surtout d'ordre qualitatif, l'islam septentrional, profondément intégré à la culture dominante du Saint Nord, étant très différent de celui du Sud et très politisé, à l'encontre de ce dernier. Cette situation résulte de l'histoire: la religion musulmane s'est infiltrée, en effet, en zone sahélienne depuis plusieurs siècles, marquant la culture des sociétés intégrées au réseau commercial transsaharien. En outre, nous l'avons vu, cette région a connu, au début du siècle dernier, une "révolution islamique" à caractère fondamentaliste et prosélyte (le jihad), débouchant sur l'instauration d'un vaste empire unifié contrôlé par une aristocratie de sultans et d'émirs -issue de ce mouvement: le sultanat de Sokoto. Si ce "jihad" a échoué à développer son expansion en zone côtière, il a étendu son emprise sur une large partie de l'aire septentrionale actuelle du pays, à l'exception d'îlots de résistance "païens" dispersés au sein de celle-ci et dans la vallée du Niger et de la Bénué.

Dans le Sud, au contraire, après l'échec du nouvel émirat d'Ilorin à conquérir le pays yoruba, la religion islamique s'est répandue au sein de celui-ci de manière toute pacifique, par le truchement de commerçants septentrionaux. Simultanément, le christianisme, porté notamment par d'anciens esclaves convertis en exil à cette religion, s'est infiltré à partir de la côte, les deux religions "importées" progressant simultanément en pays yoruba aux dépens des cultes ancestraux.

Durant la période coloniale, l'administration britannique du Nord a favorisé l'islam septentrional en tant que soutien à l'ordre aristocratique issu du jihad et marqué par son caractère fondamentaliste, politique et culturel (impact de la culture nomade peul). Elle a écarté les missions des aires islamisées, les parquant dans les ghettos païens ou les quartiers d'immigrés des villes (sabon gari). Dans le Sud, au contraire, le christianisme, porté par les nouvelles élites produites par les écoles des missions, s'est développé rapidement, favorisant les courants nationalistes. A l'heure des indépendances, le colonisateur a renforcé sa politique d'isolement des masses septentrionales et de soutien à l'aristocratie du Nord, en utilisant l'islam en guise d'idéologie. Les stratégies hégémonistes du NPC ont été perçues au Sud comme un 
retour à l'ère du jihad ${ }^{13}$. Par contre, les populations méridionales ont fait prévaloir leurs identités ethniques sur leurs identités confessionnelles, les musulmans méridionaux s'opposant à leurs coreligionnaires sur ce plan.

Sous le régime militaire suivant, la religion a cessé d'être un instrument de mobilisation. La propagande biafraise dépeignant la sécession de l'Eastern Region comme un combat entre l'islam et le christianisme était abusive, le parti fédéral, dirigé par un chrétien, mobilisant les adeptes de tous les cultes, indifféremment. L'ère des conflits politico-confessionnels semblait close. D'où la surprise causée par sa réémergence en pleins travaux de la nouvelle Assemblée constituante réunie en 1978, sous la forme d'une "affaire de la shari'a" provoquée par un parti de musulmans exigeant l'introduction dans le texte constitutionnel de mesures favorables au système judiciaire islamique. La majorité de l'Assemblée refusa cette démarche au nom de la nécessaire laïcité d'un pays multiconfessionnel ${ }^{14}$. L'intervention du gouvernement militaire empêcha que ce débat ne brise l'unité du pays et le programme de retour à un régime civil qu'il patronnait. Les nouveaux partis politiques habilités à concourir pour les élections de 1979 durent se constituer, par ailleurs, sur des bases non confessionnelles. Les musulmans du Sud, dont le ralliement était visé par le parti proshari'a en vue de la constitution d'une formation musulmane majoritaire, n'avaient pas suivi cette stratégie. Par contre, une partie des adversaires de celle-ci se sont retrouvés dans le nouveau parti NPP Mais les débats de la seconde République ont été peu marqués par la question religieuse $\mathrm{e}^{15}$.

Celle-ci a resurgi, au contraire, avec force, sous le régime militaire de janvier 1984, dans le cadre de la vague islamiste affectant au même moment une partie importante du monde musulman, de l'Iran à l'Égypte et de l'URSS au Soudan. Des groupes islamistes activistes se sont développés dans les campus et autres établissements d'études islamiques créés depuis quelques années, infiltrant la Société des étudiants musulmans (MSS), présente dans les écoles, les lycées, les universités, le Conseil des ulamas et diverses autres institutions islamiques. Une autre organisation, d'inspiration wahhabite (yan izala), se dressait simultanément contre les confréries soufies. Réclamant une Constitution islamique, le rejet du principe de la laïcité, l'instauration d'un ordre islamique dans tous les campus, attaquant les militants chrétiens, les processions, les débits de boisson et toute trace de civilisation occidentale et chrétienne, des foules musulmanes ont causé de graves tensions, débouchant sur des violences inquiétant les milieux chrétiens, eux-mêmes mus par des tendances revivalistes animées par des sectes pentecôtistes très agressives. Le point crucial de cette double mobilisation a été les émeutes qui ont ravagé l'État de Kaduna, en mars 1987, au cours desquelles cent cinquante-cinq églises et plusieurs mosquées furent brûlées, tandis que l'on comptait vingt morts et des millions de dégâts. Simultanément, certains dirigeants musulmans, dont le shaikh Gumi, figure marquante de l'islam septentrional, énonçaient des déclarations inquiétantes pour les chrétiens, et les débats de la nouvelle Assemblée constituante élue, réunie par le régime en 1988, étaient bloqués par la ré-émergence de "l'affaire de la shari'a". Les élus musulmans de cette Assemblée condamnaient en majorité le caractère laïque du projet constitutionnel et exigeaient l'intégration du système de la shari'a dans celui-ci. A nouveau, le gouvernement militaire dut intervenir pour débloquer l'impasse résultant de ces conflits en retirant aux élus ce domaine litigieux et en édictant lui-même des textes de compromis, au risque d'être accusé de briser le processus démocratique en cours. 

nigérians de l'islam au courant de "renouveau islamique" que s'efforcent de mobiliser les activistes islamistes. Et ce mouvement s'est développé jusqu'au sein de la communauté musulmane méridionale, qui s'était jusqu'alors désolidarisée des fanatiques septentrionaux, relançant les espoirs de ceux qui misent sur la mobilisation, majoritaire à leurs yeux, de l'ensemble des musulmans du pays. Sultans et émirs, imams et ulamas, réunis sous la houlette d'un Conseil suprême des affaires islamiques (SCIA) présidé par le sultan de Sokoto et d'une Société pour la victoire de l'islam (JNI) de plus en plus actifs, ont pris la tête d'un mouvement d'affirmation islamique. Des femmes musulmanes se sont séparées de la Fédération des associations féminines pour fonder une association purement confessionnelle (Fomwan). Face à ces courants nouveaux, qui débouchent sur des provocations, des violences, des accusations de discrimination religieuse, les Églises chrétiennes, rassemblées dans le cadre d'une Association chrétienne ?u Nigeria (CAN) et diverses associations laïques, s'émeuvent et se mobilisent pour la défense de la laïcité et de leur foi, menacées d'un nouveau jihad. La tension résultant de cette confrontation s'est trouvée accentuée en 1986 par l'étrange situation créée lors de la nouvelle de l'entrée du Nigeria au sein de l'OCI et l'attitude très ambiguë $d u$ gouvernement militaire à ce sujet, celui-ci ne démentant ni n'infirmant cet événement, de sorte que Ies chrétiens l'ont soupçonné de vouloir instaurer de manière clandestine un ordre islamique. Les mesures de conciliation qu'il a alors prises pour défuser une tension débouchant sur divers affrontements de rue et de campus : nomination d'un Comité pour l'examen des implications d'une entrée du Nigeria dans l'OCI, puis d'un Comité consultatif sur les affaires religieuses (ACRA), constitué pour moitié de musulmans et de chrétiens, loin de contribuer à calmer les esprits, n'ont fait que révéler la profondeur d'un antagonisme majeur. Selon des déclarations des services fédéraux de sécurité et les analyses de certains chercheurs, cette tension serait en fait le produit de machinations de puissances ou de services secrets étrangers attachés à déstabiliser le pays du fait, notamment, de ses prises de position envers les régimes d'apartheid d'Afrique australe.

La mise en place de nouveaux partis semble devoir réduire ces tensions. Mais les deux camps chrétien et musulman se surveillent. Le premier est prompt à dénoncer toute déviation de la norme "séculariste" qu'il est parvenu à maintenir dans la Constitution, les persécutions musulmanes ou les faiblesses d'un gouvernement lui-même affaibli par le partage confessionnel du corps militaire à l'égard des activistes islamistes. Le second répond à cette attitude par des prises de position, des mesures locales, des dénonciations de pratiques discriminatoires, notamment dans les écoles méridionales, et parfois des agressions justifiées par le soupçon de provocations chrétiennes. Mais l'éventualité d'un conflit confessionnel, évoquant celle d'un chaos à la libanaise ou à la soudanaise, semble surtout de l'ordre de l'intimidation politique. Musulmans et chrétiens sont divisés, les uns et les autres, en innombrables sectes, tendances, associations, Églises ou confréries, communautés régionales ou ethniques, clientèles, dont la cohésion est relative. En outre, ils participent d'autres foyers de polarisation qui peuvent les mobiliser davantage, ou refusent de se laisser entraîner dans des aventures sectionalistes et privilégient leur appartenance nationale. Quant aux sujets qui adhèrent à une position laïque, à des cultes traditionnels ou à diverses orientations concurrentes, ils critiquent la polarisation des débats du pays sur la confrontation islamo-chrétienne engendrée par les activistes des deux camps, attribuée le plus 
souvent aux machinations de "sponsors" étrangers, et exigent du gouvernement qu'il traite la situation sur la base de la mission patriotique, donc laïque, dont il se réclame.

31 Il n'en reste pas moins que la tentative de putsch du 22 avril 1990 est intervenue dans la foulée des manifestations d'organisations chrétiennes, notamment du nord de la fédération, dirigées contre le remaniement gouvernemental de décembre 1989. Leurs protagonistes estimaient qu'elles révélaient la volonté du régime d'écarter les chrétiens de la direction des affaires du pays au profit des musulmans. La déclaration lue sur les antennes nationales par les insurgés faisait référence à cette accusation de manière très nette, en proclamant que la seule solution au complot du régime pour imposer un pouvoir musulman était la partition du pays en deux blocs correspondant au fief activiste musulman du Far North d'une part, et aux États du Sud et du Middle Belt qui refusaient de se soumettre à son hégémonie, d'autre part ${ }^{16}$.

Contre-polarisations secondaires

Les grandes configurations qui viennent d'être évoquées n'épuisent pas la capacité de la société nigériane à sécréter des espaces de conflit dont l'impact est moindre que le leur mais qui contribuent aux contradictions de cette société. Une première configuration secondaire de ce type repose sur la structure socio-économique de celleci. Elle oppose, en premier lieu, l'ensemble minoritaire, mais puissant, constitué par les principaux bénéficiaires du système de redistribution de la rente pétrolière et des investissements étrangers, dont la fortune est considérable et le mode de vie provocateur (appelés millionnaires, haves, naira power, jet society) à la grande masse des citoyens au faible revenu, frappée aujourd'hui par la crise économique et les mesures de rigueur du régime (les have nots, undertrodden ou common men). Ce clivage redouble celui, plus ancien, opposant les puissants aux humbles (talakawa, mekunnu), Perçu par les militants politiques ou syndicalistes marxistes dans la perspective d'une lutte de classe (mais les tentatives de ceux-ci pour fonder un parti de classe ont toujours échoué jusqu'ici) ${ }^{17}$, il nourrit un courant populaire latent hostile aux "pillards de la richesse nationale", propice aux grandes purges anti-corruption de certains régimes (dont les victimes sont surtout en fait des fonctionnaires ou des politiciens), et parfois à des partis de type populiste, comme le Nepu ou le PRP, qui conquit les gouvernements des États de Kano et de Kaduna en 1979. Il peut déboucher également sur des révoltes paysannes (mouvement agbekoya de 1968-1970) ou à des "émeutes de la faim", telles les manifestations anti-SAP de juin 1989, suscitées il est vrai, par des étudiants, lesquels se sentent périodiquement en position de prendre en compte les aspirations de la majorité silencieuse. A un niveau plus marginal, un sousprolétariat urbain de plus en plus important répond à une situation désespérée par des provocations débouchant sur la criminalité et la délinquance. Le champ considéré s'organise, par ailleurs, sur la base de catégories socio-économiques multiples : salariés réguliers, businessmen et contractors, market women, syndicats, paysans, etc., qui constituent autant de groupes de pression exclusivement attachés à la défense ou à la promotion de leurs intérêts.

33 Une autre configuration correspond à la division du pays en un très grand nombre de collectivités locales correspondant à des entités politiques pré-coloniales: villages, hégémonies, royaumes, émirats, cités. Celles-ci demeurent des foyers très puissants de solidarité et d'identité collective, susceptibles de briser toutes les configurations où elles s'inscrivent. C'est d'ailleurs dans ce but que le colonisateur en a fait le fondement de son régime d'indirect rule, jusqu'à l'indépendance. Elles s'organisent autour de 
monarques héréditaires relevant de dynasties anciennes ou récentes. Si les Royal Fathers, Natural Rulers ou Pères de la Nation ont perdu leurs prérogatives politiques et administratives d'antan, ils demeurent entretenus par l'État, respectés par les autorités et suivis par une part importante de leurs anciens sujets, en cas de crise. Le groupe de pression qu'ils constituent est un rempart contre les agressions des "immigrés" (settlers par opposition aux natives), de l'Administration, des politiciens ou des collectivités concurrentes. Chaque communauté historique garde en mémoire le souvenir des conflits qui l'ont opposé par le passé à ses voisines, défend ses frontières, exige une représentation dans toutes les instances extérieures, au profit des "fils du sol" et peut se montrer xénophobe à l'encontre des citoyens de 1'extérieur. La plupart correspondent à des local governments, cadres de base du système fédéral national. Ceux-ci disposent de conseils et de représentants élus. C'est en leur sein que se constitue la nouve11e génération politique promue par le gouvernement militaire. La fédération compte aujourd'hui 449 local governments. Les conflits entre ces communautés territoriales, entre natives et settlers, ou encore entre celles-ci et l'État où les forces de l'ordre portent surtout sur des questions de chefferie, de frontières, de possession des terres, de respect des coutumes locales. Ils peuvent donner lieu à des batailles rangées. Depuis peu, certaines communautés musulmanes septentrionales proclament leur territoire islamic area, se qui signifie qu'ils y interdisent la consommation d'alcool ou de porc, les manifestations religieuses chrétiennes ou idolâtres, ou qu'ils entendent imposer aux jeunes élèves ou aux infirmières des uniformes islamiques. Ces mesures soulèvent l'émotion des non-musulmans et l'appel par ceux-ci à la justice ou au gouvernement, au nom du principe constitutionnel de laïcité. Il peut en résulter de graves tensions. Par contre, dans certaines sociétés, les atteintes des prosélytes venus de l'extérieur aux rites ou traditions coutumières peuvent donner lieu à des violences. Ces positions mobilisatrices alternent toutefois avec des divisions internes, aux fondements historiques souvent lointains, tout aussi violentes, selon la conjoncture.

Une autre configuration territoriale, d'origine récente, suscite des "mobilisations confrontationnelles nouvelles". Nous voulons parler des États fédérés. Conçus de manière à briser les tendances régionalistes ou ethniques centrifuges et à démultiplier la gestion de l'État, possédant des populations souvent plus importantes que la plupart des États indépendants du continent (ceux de Kano ou d'Oyo ont plus de dix millions d'habitants), les deux dernières Constitutions leur ont concédé une autonomie administrative et politique très grande, sur le modèle des USA. Chacun d'eux a son gouverneur, élu ou coopté par l'armée, selon les régimes, son assemblée élue, en période de civilianisation, son administration, son budget, ses lois propres. Établis à l'origine sur la base de partages plus ou moins arbitraires, leur assise est loin d'être toujours solide et les demandes de démembrement ne cessent d'aff1uer. Ils sont ainsi passé du nombre de douze, en 1967, à dix-neuf en 1976 et vingt et un en 1988, sans jamais satisfaire toutes les aspirations. Toutefois, ces États ont développé depuis leur création des tendances quasi nationalistes les conduisant à privilégier leurs "indigènes" aux dépens des autres citoyens de la fédération. Cette attitude est désignée sous le sobriquet de statism. Elle se traduit par des mesures discriminatoires, des dispositions légales légitimées par la coutume locale, l'imposition de taxes ou de mesures restrictives aux settlers. Elle est renforcée par le système des quotas, ou federal character, déjà évoqué, dont le non-respect peut donner lieu à des conflits interétatiques. 

trans-territoriaux, qui peuvent regrouper des sujets appartenant à des communautés très diverses. Cette structure s'enracine dans une tradition associative ancienne, aussi bien que dans le mouvement associatif moderne, qui se traduit par une prolifération $d$ 'associations professionnelles, locales, $d$ unions, de syndicats, d'improvment unions, de clubs, de lobbies, de sociétés secrètes, confréries, Eglises ou sectes. Elle se constitue autour de grandes figures polaires, personnages illustres ou noyaux plus collégiaux, contrôlant d'immenses réseaux concurrents infiltrés dans tous les espaces collectifs existant et poursuivant des objectifs hégémoniques. La base de leur pouvoir est économique: la plupart se sont constitués à la veille de l'indépendance, pour le contrôle des économies régionales ou de la répartition des ressources nationales. Sur ces bases, ils se sont développés, dans le cadre de la vie politique de la première République, en vue du contrôle des partis politiques de cette époque et des instances exécutives régionales ou nationales. Ce sont ces clientèles qui ont manipulé les polarités ethniques, régionales, religieuses et autres, dans le but d'accroître leurs positions, engendrant ou consolidant les configurations correspondantes. C'est pourquoi on les confond souvent avec les diverses composantes de celles-ci comme avec certains partis politiques. Mais leur influence dépasse les cadres, et les efforts actuels du gouvernement militaire pour écarter la "vieille garde" politique au profit d'une "nouvelle génération" (newbreed) de politiciens responsables, étrangère aux us et coutumes qui causèrent l'effondrement des deux premiers régimes civils, suscitent le scepticisme de beaucoup, les pouvoirs prétoriens eux-mêmes échappant difficilement à leurs mailles. C'est ainsi que l'on peut tenir une lecture de la vie politique du pays en termes de mafias: Kaduna mafia, vouée à la propagation des objectifs de l'ancien dirigeant du NPC et Premier ministre du gouvernement de la Northern Region: le sardauna Ahamadu Bello ${ }^{18}$; Ikenne mafia, constituée des fidèles de l'ancien leader yoruba Obafemi Awolowo, candidat toujours malheureux à la présidence nationale ; Enugu mafia, axée sur les intérêts des disciples de l'ancien président Azikiwe, puis du leader du Biafra ; Langtang mafia ou Bida old boys, très présentes au sein de l'armée, ou Kuru mafia, formée des anciens du NIPSS de Kuru. Les membres de ces réseaux nient volontiers l'existence de ceux-ci ou leur participation, de sorte que ces clientèles fonctionnent à la manière des sociétés secrètes, sans jamais être véritablement occultes. Certains dénoncent leurs extensions internationales ou les voient comme des branches d'organisations internationales qui peuvent financer leurs entreprises.

L'anti-pôle anomique

Quelles que soient les contradictions susceptibles d'opposer entre elles les différentes constellations collectives qui viennent d'être évoquées ou de commander à leur fonctionnement interne chacune d'elles est censée se soumettre à un ordre commun. Les mobilisations qui peuvent se produire au sein de chacune s'arrêtent en principe où commence le règne de la loi nationale, dont l'État est le gardien. Or, cet ordre commun est sans cesse menacé d'implosion du fait de l'existence d'une contre-polarité négative qui le hante tout en lui étant lié comme son ombre. Cet envers, cette "autre scène" s'ordonne sur la base d'un principe de désordre fondamental définissant un lieu d'anomie ou d'anarchie interdit et fascinant. Celui-ci occupe une place essentielle dans le champ des relations sociales et l'imaginaire collectif du pays. Occultée par toutes les analyses réputées "scientifiques", exagérée, au contraire, dans le discours des médias ou des pessimistes, cette donnée ne peut être écartée d'une analyse réaliste ${ }^{19}$. 

en premier lieu, dans le dérapage périodique de la dynamique du système de polarisations concurrentes évoquées précédemment: certaines composantes des configurations en question, ou l'une de celles-ci, peuvent entrer brusquement en dissidence et placer leurs objectifs au-dessus de la légalité officielle, en s'arrogeant le maniement de la violence légitime. Tel est notamment le cas des collectivités qui ont détenu par le passé une autonomie de gestion de type national. Soumises par le régime colonial à un ordre différent, dont la nation nigériane est l'héritière, mais dans le cadre ambigu d'un fédéralisme mal défini, qui se ressent des ambivalences de l'indirect rule, il leur arrive de se souvenir du passé lorsqu'elles estiment leurs droits "héréditaires" menacés ou que l'État ne fait pas preuve de l'impartialité dont il se légitime. D'autres communautés, de formation récente, réagissent de la même manière, en se référant à un droit dévoyé. De telle positions débouchent sur des pogroms, des batailles rangées, des tensions menaçant de déboucher sur une "guerre civile", mettant en cause l'ordre public et l'autorité de l'État: conflits ethniques, régionaux, religieux, de classe, de communautés locales, etc., jalonnant le champ de l'événement, sont toujours susceptibles de déboucher sur ce type de déviation. Celui-ci sert d'ailleurs fréquemment d'alibi aux auteurs de coups d'État, lesquels parent un acte fondamentalement illégal d'une autre légitimité, fondée sur un devoir sacré de rétablir l'ordre menacé par la défaillance de leurs prédécesseurs ou les menées subversives des fauteurs de troubles. Réciproquement, les États mis en cause peuvent se prétendre légitimés à traiter les comploteurs avec la dernière sévérité, même si eux-mêmes sont nés d'un acte tout aussi illégal.

Un pas de plus dans la voie de l'anomie est franchi par les "foules en colère" qui s'abandonnent périodiquement à des émeutes débouchant sur des lynchages, des destructions, des batailles rangées avec les forces de i'ordre, à l'occasion de faits divers souvent insignifiants. Ces violences improvisées s'inscrivent dans un climat de rumeurs et de soupçons à l'encontre d'un État jugé défaillant ou coupable d'utiliser illégalement la force à des fins privées. En ce cas, la foule se dresse pour rétablir un ordre dont il n'est plus de ce fait le garant légitime. Elle entend donc défendre la légalité, en raison d'une aperception toute partiale de la légitimité bafouée et des défaillances de la force publique, première cible de ses débordements, compte tenu du comportement souvent ambigu de certains de ses agents, accusés de rançonner les citoyens et de protéger les criminels, ou d'avoir partie liée avec eux. Il s'agit donc avant tout de défis à l'État, mais pour exiger une restauration de celui-ci dans ses véritables fonctions, la foule ne "prenant la loi entre ses mains", selon l'expression consacrée, que dans la mesure où elle a glissé des siennes. Un cas particulier et préoccupant est celui des lynchs de suspects de vol, de meurtre ou de sorcellerie par les mêmes foules en colère, qui se sont développés depuis quelques années, en réponse à l'extension de la criminalité. Toutes spontanées qu'elles soient, ces manifestations de justice populaire ou instant justice ${ }^{20}$, ont pris la forme d'un acte rituel, les victimes étant arrosées d'essence et brûlées devant tous. Les auteurs de telles atrocités ne se sentent généralement pas coupables, et les autorités se gardent d'intervenir, de peur de subir le même sort, ou éprouvent des difficultés considérables à trouver des témoins ou à sévir après coup. L'anarchie se pare des atours du droit, en inversant la position des coupables aux dépens de l'État fautif ${ }^{21}$. 

les militaires responsables considérant leurs actes subversifs comme légitimés par les abus de l'État en place, l'approbation du public, la gravité d'une situation susceptible, à leurs yeux, de conduire le pays au chaos. L'armée estime par ailleurs détenir un droit légitime de sauvegarde de l'unité nationale, lequel constitue sa mission fondamentale, même lorsqu'il s'exerce contre la Constitution et les autorités établies. Les lois exceptionnelles qu'ils édictent ne sont que provisoires, leur gestion débouchant sur un retour à l'ordre interrompu. En fait, cette position ne consacre que les coups d'État réussis. Malheur aux militaires qui échouent dans leurs tentatives rédemptrices, généralement jugés pour félons et rapidement exécutés par leurs compagnons de la veille, comme les assassins du général Murtala Mohammed, en 1976, le général Vatsa en décembre 1985, ou les conjurés du 22 avril, en juillet 1990.

ces comportements demeurent toutefois exceptionnels dans leur principe et toujours fondés sur quelque argument de légitimité. Tel n'est pas le cas d'une autre série d'actes illégaux commis par des individus dans la poursuite de leurs intérêts propres. L'une des manifestations les plus répandues d'un tel comportement est la sorcellerie, qui a toujours témoigné, bien avant l'ère moderne, de l'existence de tendances individualistes dressées contre l'ordre public. Cette pratique est considérée aujourd'hui comme en expansion, du fait du déclin des institutions traditionnelles censées la contrôler et du refus de l'appareil judiciaire moderne et de la loi officielle de prendre en considération les plaintes de ses victimes, ce qui revient à protéger les sorciers. Une large part de la vie occulte de la société considérée est occupée par le combat incessant de la société et de ces derniers, lequel débouche souvent sur des actes d'instant justice dont les auteurs s'estiment ainsi légitimés à rompre avec un ordre aveugle fonctionnant au profit du désordre. A ce champ particulier s'ajoute celui de la magie ou juju, qui couvre un vaste domaine d'application, de la thérapie populaire à la magie noire ou au maraboutage. Cette pratique est utilisée par les honnêtes gens pour se prémunir des criminels, les politiciens pour accéder au pouvoir, les criminels pour être invulnérables ou invisibles, les sujets avides de gains rapides pour réaliser leurs rêves. A côté de l'appel d'un général Obasanjo, ancien chef d'État, à utiliser ce black power contre l'Afrique du sud ${ }^{22}$, ce type d'activité débouche couramment sur des actes criminels, tels les meurtres rituels, ingrédients essentiels des rites de making money. En l'occurrence, des sujets quelconques ou choisis pour certaines de leurs caractéristiques sont kidnappés, immolés et dépecés de façon à pouvoir utiliser leurs organes pour confectionner des charmes. Des vagues de criminalité de ce type se produisent périodiquement, comme celle qui vient d'affecter le pays ibira (État de Kwara), en 1988-1989.

De tels débordements ne sont cependant que l'écume d'un courant de criminalité, de délinquance, $d$ 'indiscipline qui affecte toute la société à des degrés divers, à commencer par le milieu d'underground qui s'est développé depuis le boom pétrolier dans les grandes métropoles du pays. Celui-ci s'est organisé autour de la contrebande, du trafic de drogue et de devises, du vol, de la piraterie, de la prostitution. I1 devient de plus en plus agressif et audacieux, et cultive une culture de défi anarchique qui va jusqu'aux provocations publiques, au pied du poteau d'exécution. Les agissements de ces desperados entretiennent un climat de terreur qui justifie, par contrecoup, le lynchage de suspects, et entretient un sentiment de fin de l'ordre, étayé par la dénonciation de collusions entre bandits et membres de la force publique. A un autre niveau, peu de 
citoyens nigérians échappent à l'accusation des dirigeants, leaders religieux et autres censeurs ou réformateurs d'être corrompus et indisciplinés. Les libertés avec la loi s'étendent de la contrebande, du marché noir, du bakchich généralisé, notamment dans la fonction publique, sujette à de grandes purges périodiques, des viols de femmes, d'abus sexuels d'enseignants, au prestige dont jouissent quelques bandits d'honneur, comme le célèbre Anini the Law, qui prétendit opposer sa loi à celle d'une justice dévoyée. Ce climat généralisé a donné lieu à l'expression désabusée "C'est le Nigeria", et à un sentiment de crépuscule des lois auquel prétendent répondre les appels à un retour à Dieu des leaders religieux et autres leaders de pensée ou les campagnes périodiques des derniers gouvernements nigérians à une régénération morale du pays : Révolution éthique du président Shagari, Guerre contre l'indiscipline (WAI) du général Buhari, Mobilisation de masse pour le redressement économique, l'autosuffisance et la justice sociale (Manser) du général Babangida. Dans l'imaginaire traditionnel nigérian, toutefois, contrairement aux traditions exorcistes des religions "importées" ou de la culture occidentale, sur laquelle repose le système légal du pays, l'ordre et le désordre ne s'excluent pas mais coexistent éternellement et négocient entre eux. Les débordements du second seraient dus à l'abandon des processus de régulation par lesquels les ancêtres maintenaient un ordre ambigu, en équilibre instable, en négociant sans cesse la paix, la justice et la survie du groupe. D'où les appels incessants à un retour aux sources auxquels répondent ceux des activistes islamistes ou revivalistes chrétiens appelant à trancher définitivement entre le bien et le mal, quitte à projeter celui-ci sur quelque victime émissaire. Mais la tradition ancestrale ignorait l'individu isolé et toute source de droit étrangère au surnaturel. C'est pourquoi les commentaires qui considèrent la situation actuelle comme la rançon d'une ouverture à une civilisation qui fait prévaloir le sujet sur la collectivité et le droit des hommes sur ceux des dieux ou des ancêtres recueillent autant de crédit. De ce fait, les éruptions de violence transcendent le cadre de la pure anomie pour revêtir la sacralité des grands moments fondateurs que commémorent mythes et rites, lesquels sont toujours fondés sur une rupture d'interdit originelle. Face au défi qu'elles représentent, l'État moderne, ainsi mis en procès, est contraint de réexaminer les fondements de sa légitimité et de s'amender, sous peine d'entraîner le pays avec lui dans un chaos définitif.

Conclusion

La société considérée, qui compte, rappelons-le, un tiers de la population du souscontinent noir, n'entre dans aucun des schémas qui prétendent rendre compte des sociétés nationales en tant qu ensembles clos, cohérents, consensuels, mais souvent "inachevés" dont l'édification serait l'oeuvre d'un État intervenant sur une table rase constituée des ruines d'ensembles brisés par la colonisation et d'une masse de citoyens dans l'attente de ses initiatives ou tentés par telle ou telle force centrifuge dont il convient de briser l'emprise. Cette société est plus complexe, dynamique, mouvante, multipolaire, pluridimensionnelle que ne le présupposent de tels schémas. Chacun de ses membres s'y inscrit à la fois, simultanément et alternativement, dans le cadre de plusieurs espaces collectifs organisés chacun sur la base d'une polarisation distincte commandant à sa dynamique. Ces diverses configurations de rapports sociaux ne forment pas une mosaïque de blocs juxtaposés. Elles s'ordonnent sur un fond magmatique ${ }^{23}$ selon un dispositif kaléidoscopique, chaque sujet se trouvant sollicité par ses diverses appartenances ou identités à la fois, sans pouvoir échapper aux déchirements subséquents. Des conjonctures momentanées peuvent l'inciter à privilégier provisoirement l'une ou l'autre et à se mobiliser en conséquence, fut-ce 
jusqu'au sacrifice suprême. Il doit alors trancher entre les solidarités qui le sollicitent. A chaque restructuration, il doit obéir aux lois de la configuration dominante, aux dépens de celles des autres configurations. Ces mouvements entretiennent un jeu de politisation/dépolitisation autorisant à la fois les multipolarités et les mobilisations univoques et contradictoires. La polarité nationale, que l'État s'efforce de faire prévaloir sur les autres, possède un double statut ambigu de champ dominant, dont le règne requiert la dépolitisation progressive de tous les autres et de configuration alternative susceptible de se voir concurrencée par celles-ci ou l'une d'elles. Mais ces courants sont réversibles et toutes les analyses fondées sur l'opposition du processus national et d'une polarité centrifuge unique ne peuvent qu'échouer. Car les contradictions de ces sociétés ne signifient pas qu'elles n'ont pas de réalité, ni que les alternatives soient viables. Loin de toujours gérer la construction nationale d'une position rigoureuse et exclusive, l'État doit mener une stratégie empirique visant à jouer du clavier réel des possibilités que lui offre l'agencement de la société, même s'il oppose à cette pratique des dénis trop souvent pris pour argent comptant.

En ce qui concerne la société nigériane, celle-ci a cherché à consolider cette démarche en l'inscrivant dans le cadre d'un dispositif fédéral à trois détentes autorisant une certaine démultiplication du pouvoir de l'État et la conciliation de polarités divergentes.

Mais aucune formule à base territoriale ou privilégiant une polarité donnée ne peut satisfaire toutes les exigences d'une société pluridimensionnelle. Si la pratique du federal character ${ }^{24}$ satisfait certaines minorités, elle contrarie d'autres intérêts, et toute politique de promotion basée sur le mérite. C'est pourquoi le régime actuel développe aujourd'hui une autre conception du fédéralisme, dégagée des cadres géopolitiques, visant à concilier divers intérêts en présence (discours constitutionnel du 3 mai 1989). On peut s'interroger sur les applications pratiques de cette nouvelle formule. Les mêmes difficultés se retrouvent dans le choix des dispositifs de représentation des citoyens élaborés dans le cadre des dernières Constitutions et dans l'appel du président Babangida à une formule de démocratie "nigériane" inspirée des traditions du passé (discours du 24 juillet 1990).

Nous avons vu que l'un des régulateurs non reconnu mais efficace de la pratique sociale nigériane était constitué par les explosions périodiques de violence venant contrarier l'ordre public, mais aussi signifier à la société et à l'État que des bornes venaient d'être franchies, au-delà desquelles s'ouvrait le chaos, menace toujours latente dans l'imaginaire social, et toujours fondatrice. Par contre, le mouvement national est menacé par les mobilisations concurrentes de certains ensembles partiels débouchant sur des positions centrifuges de type nationalitaire qui ont failli plusieurs fois mettre en cause la survie du pays. Après les polarisations ethniques ou régionalistes qui dominèrent cette dynamique jusqu'ici (et qui ont resurgi lors du putsch avorté d'avril 1990), c'est la configuration agonistique interconfessionnelle qui semble aujourd'hui le plus en position de menacer l'unité de la nation, comme celle d'autres pays en proie au fondamentalisme islamique (le coup précité $\mathrm{s}$ inscrivait aussi dans cette problématique). Cependant, la texture même de la société nigériane contrarie de telles orientations, dans la mesure où ses membres ne s'inscrivent jamais exclusivement dans aucun champ de polarisation se proposant comme alternative unique au projet national et où aucune des configurations concurrentes qui se manifestent au sein du magma national n'est totalement close ou figée. En outre, aucune des composantes qui s'y 
dessinent n'est totalement cohérente, de sorte que les mobilisations qu'elles commandent ne peuvent généralement pas déboucher sur des constructions durables passées les grandes exaltations initiales. Ici encore, la violence peut jouer un rôle régulateur. Par contre, un régime averti et habile peut empiriquement "défuser" les tensions, les déplacer, les contrarier en suscitant d'autres foyers de polarisation alternatifs en vue de briser leurs élans, de contraindre les citoyens à s'interroger sur leurs solidarités ou identités, voire exaspérer les tensions, momentanément, pour jouer de la menace du chaos. Encore convient-il que le hasard ou une surveillance sévère désarment à temps ceux qui, au sein de l'armée, penchent pour des positions moins souples.

La nouvelle expérience de transition à un régime démocratique dans laquelle s'est engagé le régime du général Babangida, et qui doit déboucher en octobre 1992 sur une troisième république, laquelle devrait être expurgée des pesanteurs qui provoquèrent l'échec des deux précédentes, est censée ouvrir une nouvelle dynamique nationale. La gestation de ce nouveau régime s'effectue à coup de décrets de "civilianisation" progressive et de coupures drastiques : confiscation de la Constitution à l'Assemblée constituante élue pour la rédiger, pour cause de confrontations interconfessionnelles à fort relent politicien (affaire de la shari'a), éviction de l'ancienne classe politique, dissolution des partis qui s'étaient constitués après la levée du ban sur les activités politiciennes, mise à l'écart des fonctionnaires du processus électoral, imposition d'un système bipartisan dont les deux composantes ont dû se constituer autour de programmes concoctés par le gouvernement militaire, surveillance des groupes et éléments jugés subversifs, etc. Les contestations éventuelles se trouvent également brisées par la menace d'arrestations individuelles, l'interdit de rassemblements "subversifs" et surtout la crainte de voir l'armée renoncer à son projet sous le prétexte que les conditions ne seraient pas réunies pour un succès de la nouvelle démocratie. D'ores et déjà, cependant, on a pu constater que la "Nouvelle génération" (newbreed) politique qui est censée se substituer à la vieille classe politique rejetée n'échappe pas aux pesanteurs décrites précédemment, bien qu'elle se recrute massivement au sein de la même strate socio-économique: la naira society. En outre, les remaniements intérieurs de l'armée et surtout la tentative de putsch du 22 avril 1990 ont révélé la fragilité de ce support essentiel de la nouvelle cohésion nationale. Si la hausse actuelle des prix du pétrole, consécutive à la tension provoquée par l'invasion irakienne du Koweït, offre au régime des ressources inattendues en allégeant le fardeau de la crise économique et en gonflant le volume du "gâteau national" que les nouveaux politiciens auront à partager, la longue marche qui doit conduire à la troisième république sera sans doute jalonnée de fortes tensions. Et il faudra au général Babangida exercer toutes les ressources de l'habileté manoeuvrière qui lui a valu le surnom de Maradona pour la conduire à terme, s'il y parvient. Quant au sort du nouveau régime, il est trop tôt pour l'évaluer. 


\section{NOTES}

1. Les données sur lesquelles repose cette esquisse ont été recueillies depuis une trentaine d'années sur le terrain et dans de nombreux ouvrages, articles ou documents concernant ce pays, ainsi que dans la presse, très importante, de celui-ci.

2. Les évaluations démographiques actuelles se fondent sur le dernier recensement officiel, qui a eu lieu en 1963. les chiffres de celui-ci ont été contestés. Un autre recensement, qui a eu lieu en 1973, a été écarté par le régime militaire de 1975 du fait du caractère explosif des résultats. Un nouveau recensement est prévu pour 1992. Mais le président de la commission chargée de le réaliser a déclaré que certaines données, notamment ethniques et religieuses, en seraient volontairement exclues de crainte de susciter de nouvelles tensions incompatibles avec l'oeuvre de mobilisation nationale, à une période cruciale de la vie du pays.

3. B. Ahinyemi, Foreign policy and federalism. The Nigerian experience, Lagos, Macmillan Nigeria publ., 1986 ; O. Aluho, Essays in Nigeria foreign politics, London, G. Allen and Unwinn, 1981 - E. Babatope, The struggle for power in Nigeria, Iheja, J. West publ., 1981 - D. Bach, J. Egg, J. Philippe (dir), Nigeria, un pouvoir en puissance, Paris, Karthala, 1988 ; J. S. Coleman, Background to Nigeria, Univ. of Calif. Press, 1958 - E. Isichei, A history of Nigeria, New York, Longman, 1983 ; I. A. Gambari, Party politics and foreign policy, Ahmadu Bello Univ. Press, 1980 ; G. Nicolas, Évolution du système fédéral nigérian, Paris, Le mois en Afrique, juin, juillet 1980 - G. Nicolas, le Défi nigérian. Gestation d'une puissance régionale, Pans, Relations internationales, $n^{\circ} 34$, été 1981 ; G. Nicolas, Modèle fédéral et réalité nationale africaine, Le mois en Afrique, juin juillet 1985 ; G. Nicolas, Dynamique géopolitique de la fédération nigériane, Paris, Hérodote, $n^{\circ} 46$, 4ème trim. 1987 ; G. Nicolas, Les nations à polarisation variable : le cas nigérian, in E. Terray (dir.), l'État contemporain en Afrique, Paris, L'harmattan, 1987 ; B. O. Nwabueze, A Institutional history of Nigeria, USA, Longman, 1982 ; J. Wayas, Nigeria's leadership role in Africa, London, The Macmillan press ltd, 1979 - G. Williams, State and society in Nigeria, Idanre, Afrografrika, 1980.

4. Guidance : anglicisme très utilisé pour signifier le rôle de grand frère du Nigeria.

5. A Ademoyega, Why we struck. The story of the first nigerian group coup, Evan Br ltd, 1981 ; Ola Balogun, The tragic years. Nigeria in crisis, Eth. Publ. Corp., Benin city Nigeria, 1973 ; Ben Gbulie, Nigeria's five majors, Onitsha, Africana educ publ, Itd Fr. Forsyth, Emeka, Ibadan, Spectrum boohs, 1982 ; A. A. Madiebo, The nigerian revolution and the civil war, Enugu, Fourth Dim publ, 1980 ; D. J. M Muffett, Let truth be told, Íaria, Huda huda publ, 1982 ; A Nwanko, Nigeria : The challenge of Biafra, Enugu, Fourth dim. Publ., 1972 - O. Obasango, My mmand, London, Heineman, 1980 ; Obasungo, Nzeogwu, Ibadan, Spectrum books ltd, 1987.

6. Le général Babangida a proclamé, à son avènement au pouvoir, en août 1985, son attachement aux principes des Droits de l'homme et diverses mesures visant à la promotion de ceux-ci. L'attribution du prix Nobel à l'écrivain Wole Soyinka a été perçue comme la sanction de celte orientation nouvelle, tout autant que celle des qualités de son oeuvre. Le lauréat devait peu après être nommé à la tête d'un organisme chargé de la discipline des conducteurs routiers.

7. R. Anifowose, Violence and politics in Nigeria. The tiv and yoruba experiences, Enugu, Noh publ., 1982 ; J.S. Coleman, op. cit. ; D. laitin. Hegemony and culture. Politics 
and religious change among the Yoruba, Chicago, Univ. Ot Chicago Press, 1986 ; G. Nicolas, Crise de l'État et affirmation ethnique en Afrique noire contemporaine, Paris, Revue française de science politique, XXII, oct. 1972 ; G. Nicolas, Stratégies ethniques et instruction nationale au Nigeria, in J. P. Chrétien et G. Prunier (dir.), Les ethnies ont une histoire, Paris, Karthala, 1989 ; O. Nnoli, Ethnic politics in Nigeria, Enugu, Fourth Dim publ, 1978 - R Sklar, Nigerian political parties : power in an emergent african nation, Princeton Univ. Press, 1963 ; A Nwanko, The igbo leadership and the future of Nigeria, Enugu, Fourth Dim publ., 1986 ; G Williams, State and society in Nigeria, Idanre, Afrografrika publ., 1980.

8. Le terme Wazobia juxtapose les trois verbes signifiant aller dans les trois langues yoruba, hausa et igbo.

9. Les minorités côtières de l'ancienne région de l'Est, qui se sont mobilisées sous la première république contre l'hégémonisme de leur grand frère" igbo et du parti NCNC, sont couramment désignées sous l'appellation COR, qui juxtapose les initiales des trois provinces où elles se situent : Calabar, Ogoja et Rivers. Les territoires de celles-ci correspondent aujourd'hui aux trois Etats fédérés de Cmss Riuer, Ahwa Ibom et Riuers, distincts des deux Etats igbos d'Anambra el Imo.

10. La république du Biafra, proclamée par le gouvernement militaire de l'Eastern Region le 30 mai 1967 (jour anniversaire des pogroms anti-lgbos du "Nord" de 1966) correspondait au territoire de cette entité politico-administrative. Son nom était emprunté à celui de la baie qui bordait les rives atlantiques de celle-ci, dont les origines sont incertaines. Son drapeau associait trois couleurs : le rouge, symbole du sang des martyrs des pogroms antérieurs, le noir, symbole de deuil pour ces martyrs et le vert, symbole du progrès, et un soleil, symbole d'émergence. Son espoir résidait dans le contrôle des nouveaux gisements de pétrole découverts sur ses rives, en territoire "minoritaire". Elle a capitulé le 12 janvier 1970, après le départ en exil du colonel Ojukwu, son animateur.

11. L'ethnie hausa a intégré en son sein, depuis le jihad du siècle dernier les éléments peuls (fulani) qui ont assuré le triomphe de celui-ci et formé la nouvelle aristocratie du sultanat de Sokoto. D'où l'expression courante de Fulani-hausa pour désigner l'ensemble composite résultant de celte fusion. Ce fait illustre la complexité du cadre ethnique.

12. Abdurahman Doi, Islam in Nigeria, Zaria, Gashiya ltd, 1984 ; E. C. Amucheozi, Church and politics in Eastern Nigeria, 1945-1966, Lagos, Macmillan 1986 ; Y Bala Usman, The manipulation of religion in Nigeria, Kaduna, Vanguard press, 1987 ; V Chukwulozie, Muslim-christian dialogue in Nigeria, Ibadan, Day star press, 1966 ; Federal Republic of Nigeria : Report of tribunal of inquiry on Kano disturbances, Lagos, Fed Gov. Press, 1981 ; E. Isichei (ed.), Varieties of christian experience in Nigeria, London Macmillan, 1982 ; D. laitin, Hegemony and culture, op. cit. ; G. Nicolas, Métamorphoses de l'islam nigérian, Paris, Le mois en Afrique, août-sept et oct-nov. 1984 ; G. Nicolas, Guerre sainte à Kano, Paris, Politique africaine, I, 4, nov. 1981 ; G. Nicolas, Le carrefour géopolitique nigérian et les axes islamiques sahélo-guinéens, Paris, Hérodote, 4ème trim. 1984 ; Nasir B Zahdreen, The maitatsine saga, Zaria, Huda huda (ed), 1988 ; B. Clarke and Ian Linden, Islam in modern Nigeria, Mainz, KaiserGrunewald, 1984 ; Syed Khalid Rashid (ed.), Islamic law in Nigeria, Lagos, Islamic Bureau, 1986.

13. Le corps du Premier ministre général Abubakar Tafewa Balewa, membre du NPC, aurait été retrouvé au lieu même où échoua, au siècle dernier, la dernière tentative des 
jihadistes septentrionaux d'atteindre l'océan, les yeux crevés et la face tournée vers la mer. Ce fait témoigne de la force de la vision sudiste de l'hégémonie du NPC (conduit par un descendant du leader du jihad, également victime des insurgés ) comme regain de celui-ci et tentation continue de "tremper le Coran dans la mer", selon une expression consacrée. Cf. J.M. Mullett, Let truth be told, The coups d'Etat of 1966, aria, Huda huda publ Cy 1982.

14. Les dix États fédérés issus du démembrement à l'ancienne Northern Region disposent d'un appareil juridique "islamique" (tribunaux de la shari'a), en sus de l'appareil de droit commun ou coutumier. Ces tribunaux ont compétence pour des questions de droit civil personnel. Les Constitutions de 1979 et 1989 ont intégré cette structure, combattue par les tenants d'une sécularité absolue de l'État nigérian, ouvrant la possibilité de l'étendre aux États méridionaux qui le souhaiteraient, dans le cadre de leurs lois propres et pour leurs seuls membres adeptes de l'islam. Les revendications du parti pro-chari'a concernaient surtout d'insertion de cet appareil juridique dans le cadre de la Cour suprême de la fédération et le choix des juges chargés des jugements en appel des décisions des tribunaux des États. Ce sont ces exigences qui ont été rejetées, au nom de la nécessaire sécularité d'un Etat pluri-confessionnel.

15. Notons toutefois les massacres et destructions engendrées par le jihad d'une secte marginale mais particulièrement agressive, dirigée par un marabout d'origine camerounaise : Muhammadu Manua, dit Maitatsine. Cette secte, qui aurait rassemblé près de dix mille adeptes, lesquels se recrutaient dans les milieux de désespérés du Sahel affectés par la sécheresse, la crise économique, l'été rural, a mis à feu et à sang plusieurs métropoles septentrionales, dont Knno (décembre 1980), Maiduguri et Knduna (a :tobre 1982j, Yola ((éurirr 1984) et Gombe (avril 1985), inquiétant fortement le gouvernement fédéral et les États voisins, qui redoutaient l'expansion du mouvement. A chaque fois, ses tentatives subversives ont été brisées, au prix d'une répression massive, par l'armée, qui s'est substituée à la police incapable de les contrôler et des groupes de vigiles "orthodoxes" tout aussi violents que les fanatiques. Cf. notamment G. Nicolas : Guerre sainte à Kano, Paris, Politique africaine, $\mathrm{n}^{\circ} 1,4$, nov. 1981 ; Nasir B Zahradeen, The maitatsine saga, op cit.

16. Dans leur proclamation diffusée sur les ondes nationales, les officiers putschistes annonçaient que "les Etats musulmans du Nord avaient été détachés du Nigeria et que les gens originaires du Nord et travaillant dans le Sud avaient une semaine pour regagner leur territoire", Le Monde, 24/4/90. Voir aussi Jeune Afrique ${ }^{\circ} 1531 \mathrm{du}$ 7/5/90, African Guardian du 4/6/90, West Africa du 9/5/90.

17. Un labour party s'est constitué au début de 1989 , sous l'impulsion du Nigerian Labour Congress (NLC), fédération syndicale officielle et unique des travailleurs. Le programme de cette formation était idéologiquement très modéré, du fait du partage du NLC entre fonctions "progressiste" et "démocrate" et de la crainte d'un rejet par la commission charge de sélectionner les deux partis admis à concourir lors des élections futures et par un gouvernement militaire qui a manifesté à plusieurs reprises son hostilité à tout courant extrémiste. Interdit par la suite, ses militants se sont regroupés au sein du parti social-démocrate (SDP).

18. Bala J. Takaya and Sonni Gwanle Tyoden, The Kaduna mafia. A study of the rise, development and consolidation of a nigerinn power elite, Jos, Jos Univ. Press ltd, 1987 ; Rufai Ibrahim, Baban gida. Beware of Kaduna mafia, Sunday New Nigerian, I/II/85. 19. R. Anifowose, Violence and politics, op cit Asikpo Essien Ibok, ed., Political repression and assassination, Zaria, Caskiya Corpo Ltd, 1983 ; Y. Bala Usman, Political 
repression in Nigeria, Zaria, Gaskiya Corpo Ltd, 1982 ; G. Nicolas, Cette loi qu'on prend entre ses mains. La pratique de l'instant justice sous la seconde République nigériane, Paris, Droit et Cultures, $\mathrm{n}^{\circ}$ 7, 1984.

20. Cf. G. Nicolas, "Cette loi qu'on prend entre ses mains. La pratique de l'instant justice sous la seconde République nigériane", in Droit et Cultures, Paris, n 7, 1984.

21. Les Elections de l'été 1983 ont été marquées par des violences extrêmes, comportant notamment le lynchage par crémation de membres du parti national par des partisans de l'UPN, dans plusieurs Etats de l'ouest. Ces violences, qui répondaient à la menace de plusieurs leaders de ce dernier parti de "prendre la loi entre (leurs) mains" au cas où ces élections seraient truquées, répétaient celles qui ont assombri les dernières élections de la première République, en1964-1965.

22. Cf. Daily Times, 17 /6/86 National Concord, 20/6/86.

23. La théorie du magma a été forgée par Cornélius Castoriadis pour rendre compte d'un type d'organisation présenté par les "significations imaginaires-sociales" dans une société donnée : "Un magma contient des ensembles - et même un nombre indéfini d'ensembles - mais n'est pas réductible à des ensembles ou à des systèmes d'ensembles. Il ne peut être reconstruit analytiquement, à savoir au moyen de catégories ou d'organisations ensemblistes (C. Castoriadis, Domaines de l'homme. Les carrefours du labyrinthe II, Paris, Seuil 1978, p. 230-231).

24. NIPSS : Federal character and national integration in Nigeria, Jos, Salama Press ltd, 1987.

\section{RÉSUMÉS}

La crise actuelle des pays " en développement " signe l'échec des perspectives qui ont guidé jusqu'ici analystes et gestionnaires, dont les schémas glissent sur une réalité plus complexe, mouvante, multidimensionnelle, contradictoire qu'ils ne l'envisagent. Les membres des sociétés concernées sont mus par des polarités mobilisatrices diverses qui les sollicitent alternativement et simultanément, se contrarient, entretiennent une dynamique de tension. Le géant nigérian illustre cette situation : une polarisation nationale (fédérale) dominante se trouve confrontée à des contre-polarités régionales, ethniques, confessionnelles... multiples, sur un fond d'antipolarisation anomique. Dans ce champ social mouvant, soumis à des transferts et amalgames perpétuels, la violence fait fonction de régulateur. Les stratéges politiques manipulent polarisations, mobilisations et violence, transférent celle-ci d'un pôle à l'autre, en jouant de la crainte du chaos. Cette société effervescente et dynamique s'accomode d'une situation qui reste méconnue des modèles d'approche réputés scientifiques, toujours désavoués par l'évènement. De nouveaux schémas d'analyse, de nouveaux concepts sont nécessaires pour appréhender le type de fait évoqué.

The actual crisis of the underdeveloped countries shows the failure of orthodox perspectives and analyses. They have no possibilities of examining a type of changing, moving, contradictory reality. In the Nigerian case, the dominating national polarisation (a federal one) is competed by counter-polarisations (regional, ethnic, confessional...) and by the anti-polarisation of anomic 
violence. The politicians manipulate these polarisations, mobilisations, and the violence to serve their interests. To understand this type of reality, new paradigms are necessary.

INDEX

Index géographique : Afrique de l'ouest, Nigéria

Mots-clés : conflits, identité

\section{AUTEUR}

GUY NICOLAS

Professeur des Universités, titulaire d'une chaire de civilisation à l'INALCO 